\title{
EFFECTS OF TIME DELAY ON FEEDBACK STABILISATION OVER SIGNAL-TO-NOISE RATIO CONSTRAINED CHANNELS
}

\author{
Julio H. Braslavsky ${ }^{*, 1}$ Richard H. Middleton ${ }^{*, 2}$ \\ James S. Freudenberg ${ }^{* *, 3}$ \\ ${ }^{*}$ Centre for Complex Dynamic Systems and Control, The \\ University of Newcastle, Callaghan NSW 2308, Australia. \\ ** Department of EECS, University of Michigan, Ann Arbor, \\ MI48109-2122 USA.
}

\begin{abstract}
The expanding integration of control and communication networks in recent years has generated an increasing interest in control problems with feedback over a communication channel. Significant research activity has concentrated on stabilisation in face of channel effects such as quantisation and data-rate limits. In a recent paper, the authors have studied the problem of feedback stabilisation over a communication channel with a constraint on the admissible signal-to-noise ratio (SNR). It has been shown therein that for a delay-free, linear time-invariant feedback loop, a SNR constraint in the feedback channel imposes fundamental limitations in the ability to achieve closed-loop stability. The present paper extends these results by introducing a time-delay in the loop, and shows that the lowest SNR required for closed-loop stability increases by a factor that may grow exponentially with the time-delay and the unstable open loop poles of the system. This result contributes to the quantification of performance tradeoffs in integrated control and communication environments. Copyright(C)2005 IFAC.
\end{abstract}

Keywords: Communication channels, Signal-to-noise ratio, Time delay, LQG control.

\section{INTRODUCTION}

This paper considers the problem of feedback stabilisation over an idealised continuous-time communication channel with a constraint on the input signalto-noise ratio (SNR). The setting is illustrated in Figure 1, in which $G(s)$ and $K(s)$ are respectively plant and controller, with $G(s)$ minimum phase but possibly unstable. The feedback channel is represented by the linear input-output relation

$$
u_{r}(t)=u_{s}(t)+n(t),
$$

where $n(t)$ is a continuous-time, zero-mean additive white Gaussian noise (AWGN) with intensity $\Phi$,

\footnotetext{
1 E-mails: Julio.Braslavsky@newcastle.edu.au

2 Richard.Middleton@newcastle.edu.au

3 E-mail: jfr@eecs.umich.edu
}

$$
\mathrm{E}\{n\}=0, \quad \mathrm{E}\left\{n^{\prime}(t) n(\tau)\right\}=\Phi \delta(t-\tau), \quad \forall t, \tau \in \mathbb{R},
$$

where $n^{\prime}$ represents the transpose of $n, \mathrm{E}\{\cdot\}$ is the expectation operator and $\delta(t)$ is the unit impulse. The channel is assumed to have a constraint on its SNR, i.e.,

$$
\frac{\left\|u_{s}\right\|_{R M S}^{2}}{\Phi}=\frac{\mathrm{E}\left\{u_{s}^{\prime}(t) u(t)\right\}}{\Phi}<\frac{\mathcal{P}}{\Phi}
$$

for some predetermined value $\mathcal{P}<0$.

This idealised model of a communication channel is common in Communications and is usually called the AWGN channel (e.g., Cover and Thomas, 1991).

A setting such as that of Figure 1 has been considered in a recent work by the authors (Braslavsky et al., 2004), in which it has been shown that there exist a fundamental constraint on the lowest admissible 


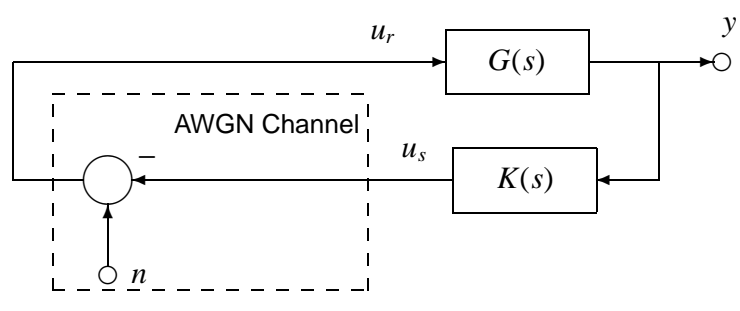

Figure 1. Feedback loop over an AWGN channel

SNR required to stabilise an unstable plant. More specifically, it has been shown that, in order to achieve closed-loop stability, the SNR (2) must satisfy the constraint

$$
\frac{\mathcal{P}}{\Phi}>\sum_{k=1}^{m} 2 \operatorname{Re}\left\{p_{k}\right\}>0,
$$

where $p_{k}, k=1,2, \ldots, m$, are the open loop unstable poles of $G(s)$.

Control over communication channels has attracted increasing interest in recent years and, in particular, a number of works have considered feedback stabilisation subject to quantisation effects and data-rate constraints (e.g., Delchamps, 1990; Wong and Brockett, 1999; Elia and Mitter, 2001; Nair and Evans, 2002; Ishii and Francis, 2003; Liberzon, 2003). The work in Braslavsky et al. (2004), although in a completely different scenario, was inspired mainly by the work in Nair and Evans (2002).

Despite the disparity in their derivations, a remarkable link can be drawn between the SNR constraint (3) and the main result in Nair and Evans (2002). Namely, if one assumes that the discrete-time system considered in Nair and Evans (2002) arises as the discretisation with sampling period $T$ of a continuous-time plant $G(s)$, then the lowest data-rate $R / T$ (bits per second) required for stabilisation must satisfy the positive lower bound constraint

$$
\frac{R}{T}>\log _{2} e \sum_{k=1}^{m} \operatorname{Re}\left\{p_{k}\right\}, \quad \text { (bits per second), }
$$

where, again, $p_{k}, k=1,2, \ldots, m$, are the open loop unstable poles of $G(s)$. On the other hand, the capacity $C$ of a continuous-time AWGN channel as in Figure 1 — assuming no bandwidth restrictions - can be made arbitrarily close to (Cover and Thomas, 1991, p. 250)

$$
C=\frac{\mathcal{P}}{2 \Phi}\left(\log _{2} e\right) \quad \text { (bits per second). }
$$

Hence, from (3), the maximum channel capacity (5) permitted by Shannon's Theorem must satisfy

$$
C>\log _{2} e \sum_{k=1}^{m} \operatorname{Re}\left\{p_{k}\right\}, \quad \text { (bits per second), }
$$

which coincides with (4) - assuming maximum capacity can be attained. This link reinforces the suggestion made in Nair et al. (2004) that such fundamental limitation is determined only by the unstable poles of the system, independently of quantisation effects, coding, decoding and control schemes.
In subsequent work (Middleton et al., 2004), the authors extended the results of Braslavsky et al. (2004) to non-minimum phase systems, and showed that the existence of non-minimum phase zeros, in addition to unstable poles, increase the lower bound (3). It was also shown in that paper that the additional term can be arbitrarily reduced by using a linear sampled-data controller with fast sampling, again recovering Nair and Evans's formula. Such recovery, however is only possible at the expense of stability robustness.

This paper extends the continuous-time results of Braslavsky et al. (2004) to plants with an input-output time-delay. Time delays are an intrinsic characteristic of non-ideal communications and pose fundamental constraints in networked control systems performance (e.g., Lian et al., 2002). The main contribution of this paper is a formula that quantifies the additional penalty imposed by a time delay on the lowest admissible SNR required to stabilise an unstable plant over the AWGN channel of Figure 1.

The rest of the paper is organised as follows: preliminaries and technical results are presented in Section 2, together with an illustrative example and some links and interpretations. All proofs are given in Section 3. Section 4 presents some concluding remarks.

\section{RESULTS}

Consider the continuous-time, single-input single output scheme of Figure 1, where the plant is given by

$$
G(s)=G_{0}(s) e^{-s \tau},
$$

where $\tau \geq 0$ is a fixed time delay, and $G_{0}(s)$ is a rational and strictly proper transfer function. For simplicity, we assume that $G_{0}(s)$ has no zeros in the closed right half of the complex plane $\mathbb{C}^{+}$.

Since the closed-loop is stable, the transmitted control signal $u_{s}$ resulting from the noise input $n$ is a stationary stochastic process with Gaussian distribution and power given by

$$
\begin{aligned}
\left\|u_{s}\right\|_{R M S}^{2} & =\left(\frac{1}{2 \pi} \int_{-\infty}^{\infty} \operatorname{trace}\left\{T(j \omega) T^{\prime}(j \omega)\right\} d \omega\right) \Phi \\
& =\|T\|_{H_{2}}^{2} \Phi,
\end{aligned}
$$

where $T(s)$ is the closed loop transfer function between $n$ and $u_{s}$,

$$
T(s)=\frac{K(s) G(s)}{1+K(s) G(s)} .
$$

We aim to find the lowest value of the SNR $\mathcal{P} / \Phi$ compatible with stability of the closed-loop, which, in view of (2) and (8), amounts to minimising the $\mathrm{H}_{2}$ norm of the transfer function $T(s)$ over the set of all internally stabilising proper LTI controllers $\mathcal{K}_{G}$.

In order to state the main result, introduce a minimal state space realisation of $G_{0}(s)$ defined by the matrices 


$$
\left[\begin{array}{ll}
A & B \\
C & 0
\end{array}\right]=\left[\begin{array}{cl}
{\left[\begin{array}{cc}
A_{1} & 0 \\
0 & A_{2}
\end{array}\right]\left[\begin{array}{l}
B_{1} \\
B_{2}
\end{array}\right]} \\
{\left[\begin{array}{cc}
C_{1} & C_{2}
\end{array}\right] \quad 0}
\end{array}\right], \quad \begin{aligned}
& A \in \mathbb{R}^{n \times n}, B \in \mathbb{R}^{n \times 1}, \\
& \text { 1×n }
\end{aligned}
$$

in which the spectrum of $A_{1}$ consists of all the poles of $G_{0}(s)$ in $\mathbb{C}^{+}$.

Introduce also the state feedback matrix

$$
F=B^{\prime} P, \quad \text { where } P=\left[\begin{array}{cc}
P_{1} & 0 \\
0 & 0
\end{array}\right] \in \mathbb{R}^{n \times n},
$$

and $P_{1}$ is the unique symmetric and positive definite solution of the minimum energy Riccati equation

$$
P_{1} A_{1}+A_{1}^{\prime} P_{1}=P_{1} B_{1} B_{1}^{\prime} P_{1} \text {. }
$$

The main result of the paper follows.

Theorem 2.1. Consider the feedback loop of Figure 1. Assume that the plant $G(s)$ is proper; has $m$ poles $p_{k}, k=1,2, \ldots, m$ and no zeros in $\mathbb{C}^{+}$, and an inputoutput time delay $\tau \geq 0$. Assume also that $K(s)$ is proper and such that the closed loop is internally asymptotically stable.

Then,

$$
\inf _{K \in \mathcal{K}_{G}}\|T\|_{H_{2}}^{2}=\sum_{k=1}^{m} 2 \operatorname{Re}\left\{p_{k}\right\}+\int_{0}^{\tau} F e^{A \sigma} B B^{\prime} e^{A^{\prime} \sigma} F^{\prime} d \sigma,
$$

where $A, B$ and $F$ are given in (10) and (11).

Together with (2) and (8), Equation (13) implies that the lowest SNR required for closed-loop stability on the system of Figure 1 is

$$
\frac{\mathcal{P}}{\Phi}>\sum_{k=0}^{m} 2 \operatorname{Re}\left\{p_{k}\right\}+\eta
$$

where the nonnegative term

$$
\eta \triangleq \int_{0}^{\tau} F e^{A \sigma} B B^{\prime} e^{A^{\prime} \sigma} F^{\prime} d \sigma \geq 0
$$

represents the cost added by the time delay with respect to the delay-free case (3). A similar expression is obtained in Mirkin and Raskin (2003, Lemma 9).

Note that although $\eta$ depends on the full matrices $A$ and $B$, only the unstable poles of the system (i.e., the eigenvalues of $A_{1}$ ) contribute to the cost, because of the modal structure of the realisation (10) and the structure of the minimum energy gain $F$ in (11).

Example 2.1. For a plant with a single unstable pole at $s=p$ we have that $A_{1}=p$ and $B_{1}=1$ are suitable matrices in the minimal realisation (10), and therefore solving (12) we obtain $F=-2 p$. Then (15) gives

$$
\eta=\int_{0}^{\tau}(2 p)^{2}\left(e^{p t}\right)^{2} d t=2 p\left(e^{2 p \tau}-1\right),
$$

and thus the SNR required for stability from (13) is

$$
\frac{\mathcal{P}}{\Phi}>2 p e^{2 p \tau}
$$

We see that the lowest admissible SNR required to stabilise this system increases by a factor that grows exponentially with the time delay in the loop.

For a plant with more than one unstable pole it seems hard to obtain a general closed-form expression for $\eta$ displaying the explicit dependence on the poles and time delay as in (16). However, we can derive a "tight" upper bound of $\eta$ (tight in the sense that the bound approaches the exact value as $\tau \rightarrow 0$ ), as shown next.

Corollary 2.2. Under the conditions of Theorem 2.1,

$$
\begin{aligned}
\inf _{K \in \mathcal{K}_{G}}\|T\|_{H_{2}}^{2} & =\left(\sum_{k=1}^{m} 2 \operatorname{Re}\left\{p_{k}\right\}\right) e^{\left\{\sum_{k=1}^{m} 2 \operatorname{Re}\left\{p_{k}\right\} \tau\right\}}-O\left(\tau^{2}\right) \\
& \leq\left(\sum_{k=1}^{m} 2 \operatorname{Re}\left\{p_{k}\right\}\right) e^{\left\{\sum_{k=1}^{m} 2 \operatorname{Re}\left\{p_{k}\right\} \tau\right\}}
\end{aligned}
$$

From (2) and (17), a sufficient condition for closedloop stabilisability can then be simply expressed as

$$
\frac{\mathcal{P}}{\Phi}>\left(\sum_{k=1}^{m} 2 \operatorname{Re}\left\{p_{k}\right\}\right) e^{\left\{\sum_{k=1}^{m} 2 \operatorname{Re}\left\{p_{k}\right\} \tau\right\}}
$$

The conservativeness of this condition reduces as $\tau^{2}$ as $\tau \rightarrow 0$. However, as will be clear from the proof of the Corollary in $\S 3.2$, the location of the poles also plays a role, as we illustrate in the following example.

Example 2.2. Suppose that the plant has two real distinct unstable poles $p_{1}, p_{2}>0$. For simplicity, take (and this is generic for two real distinct poles):

$$
A_{1}=\left[\begin{array}{cc}
p_{1} & 0 \\
0 & p_{2}
\end{array}\right] \text { and } B_{1}=\left[\begin{array}{l}
1 \\
1
\end{array}\right] \text {. }
$$

After some work, the minimum energy cost matrix $P_{1}$ can be obtained from (12) as

$$
P_{1}=\frac{2\left(p_{1}+p_{2}\right)}{\left(p_{1}-p_{2}\right)^{2}}\left[\begin{array}{cc}
p_{1}\left(p_{1}+p_{2}\right) & -2 p_{1} p_{2} \\
-2 p_{1} p_{2} & p_{2}\left(p_{1}+p_{2}\right)
\end{array}\right],
$$

and thus, the minimum energy feedback gain $F$ is

$$
F=\frac{\left(p_{1}+p_{2}\right)}{\left(p_{1}-p_{2}\right)}\left[2 p_{1}-2 p_{2}\right] \text {. }
$$

It then follows after a little algebra that for this case:

$$
\begin{gathered}
\inf _{K \in \mathcal{K}_{G}}\|T\|_{H_{2}}^{2}=2\left(p_{1}+p_{2}\right) e^{2\left(p_{1}+p_{2}\right) \tau} \\
\times \underbrace{\left[\frac{\left(p_{1} e^{-p_{1} \tau}-p_{2} e^{-p_{2} \tau}\right)^{2}+p_{1} p_{2}\left(e^{-p_{1} \tau}-e^{-p_{2} \tau}\right)^{2}}{\left(p_{1}-p_{2}\right)^{2}}\right]}_{\gamma} .
\end{gathered}
$$

Figure 2 plots the term $\gamma$ from (21) for different values of $p_{1}$ and $p_{2}$ in the interval $[0,5]$ and $\tau=0.25$ (top) and $\tau=1$ (bottom). We can see that $0 \leq \gamma \leq 1$ and also that the surface flattens down (which means that the bound (17) becomes more conservative) for larger $\tau$ and $p_{1} \approx p_{2}$. 

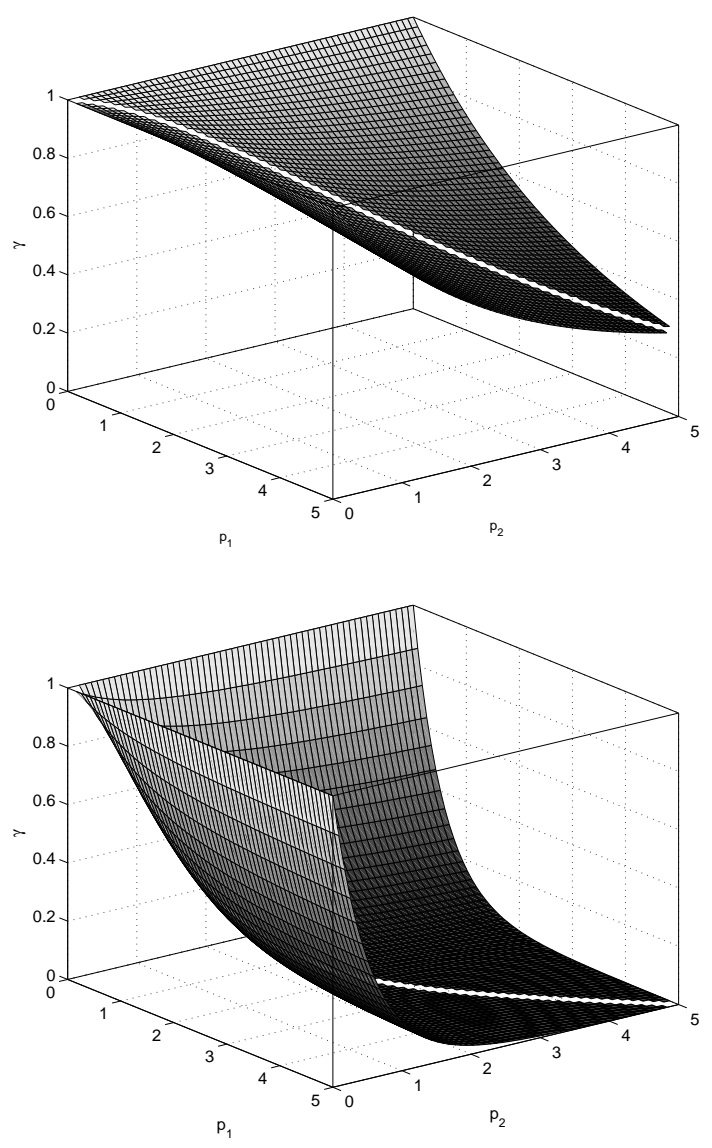

Figure 2. Plots of $\gamma$ vs. $p_{1}$ and $p_{2}$ for $\tau=0.25$ and 1

\section{PROOFS}

The key instrument to prove Theorem 2.1 is a coprime factorisation of a plant with a time delay. See Mirkin and Raskin (2003) and the references therein for more comprehensive treatments.

Lemma 3.1. Let $G(s)=G_{0}(s) e^{-s \tau}$, with $\tau \geq 0$ and $G_{0}(s)$ a strictly proper, minimum phase, but possibly unstable rational transfer function. Let $\left[\begin{array}{ll}A & B \\ C & 0\end{array}\right]$ be a minimal realisation of $G_{0}(s)$, and let $L$ be any constant matrix such that $(A-L C)$ is Hurwitz. Introduce the transfer functions

$$
\begin{gathered}
N(s)=e^{-s \tau} N_{0}(s), \\
\text { with } N_{0}(s)=C(s I-A+L C)^{-1} B, \text { and } \\
D(s)=\frac{1}{1+C(s I-A)^{-1} L} .
\end{gathered}
$$

Then, $N(s), D(s) \in H_{\infty}$ and $G(s)=N(s) / D(s)$ is a coprime factorisation of $G(s)$.

Moreover, let $F$ be a stabilising state feedback matrix such that $(A-B F)$ has the same eigenvalues as $(A-$ $L C)$. Then the transfer functions

$$
\begin{aligned}
& X(s)=F e^{A \tau}(s I-A+L C)^{-1} L \\
& Y(s)=1+ F e^{A \tau}(s I-A+L C)^{-1} B e^{-s \tau} \\
&+F\left(I-e^{-(s I-A) \tau}\right)(s I-A)^{-1} B
\end{aligned}
$$

are both in $H_{\infty}$ and satisfy the Bezout identity

$$
N(s) X(s)+D(s) Y(s)=1 .
$$

PROOF. We first show that $N(s), D(s) \in H_{\infty}$. It is obvious that $N(s) \in H_{\infty}$ since, by definition, its poles are the eigenvalues of $(A-L C)$, which is Hurwitz. On the other hand, note that we can represent $D(s)$ by the feedback loop in Figure 3, which in turn has the state space realisation

$$
\begin{aligned}
& \dot{x}=A x+L(u-y)=(A-L C) x+L u \\
& y=C x .
\end{aligned}
$$

From (25), the poles of $D(s)$ are also the eigenvalues of $(A-L C)$, and thus $D(s)$ is also in $H_{\infty}$.

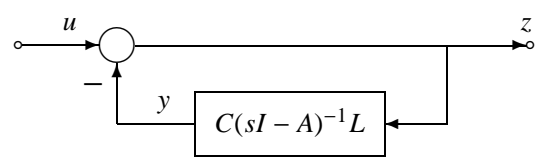

Figure 3. Feedback representation of $D(s)$

We now show that $N(s) / D(s)$ is a factorisation of $G(s)$. By using the Sherman-Morrison-Woodbury formula (Golub and Van Loan, 1996, p. 50) on the matrix ( $s I-$ $A+L C)^{-1}$ we can write

$$
\begin{aligned}
N_{0}(s) & =C(s I-A+L C)^{-1} B \\
& =C(s I-A)^{-1} B \\
-C & (s I-A)^{-1} L\left(1+C(s I-A)^{-1} L\right)^{-1} C(s I-A)^{-1} B \\
& =\left(1-\frac{C(s I-A)^{-1} L}{1+C(s I-A)^{-1} L}\right) C(s I-A)^{-1} B \\
& =\frac{C(s I-A)^{-1} B}{1+C(s I-A)^{-1} L} .
\end{aligned}
$$

Thus,

$$
\begin{aligned}
\frac{N(s)}{D(s)} & =e^{-s \tau} N_{0}(s)\left(1+C(s I-A)^{-1} L\right) \\
& =e^{-s \tau} C(s I-A)^{-1} B=G(s) .
\end{aligned}
$$

Finally, we show that $N(s)$ and $D(s)$ are coprime, that is, that there exist transfer functions $X(s), Y(s) \in H_{\infty}$ such that the Bezout identity (24) is satisfied. Consider the transfer functions $X(s)$ and $Y(s)$ defined in (22) and (23).

It is straightforward from (22) that $X(s)$ is a rational, stable and proper transfer function, and is therefore in $H_{\infty}$. On the other hand, $Y(s)$ is in $H_{\infty}$ if the term

$$
H(s) \triangleq F\left(I-e^{-(s I-A) \tau}\right)(s I-A)^{-1} B
$$

in (23) is in $H_{\infty}$. Because $H(s)$ has an impulse response $h(t)$ that vanishes outside the interval $[0, \tau)$,

$$
h(t)= \begin{cases}F e^{A t} B & \text { if } 0 \leq t<\tau \\ 0 & \text { if } t \geq \tau,\end{cases}
$$

it follows that $H(s)$ is uniformly bounded in the closed right half plane, since for $s=r e^{j \theta}$ 


$$
\begin{aligned}
\left|H\left(r e^{j \theta}\right)\right|^{2} & =\left|\int_{0}^{\tau} F e^{A t} B e^{-r t e^{j \theta}} d t\right|^{2} \\
& \leq \int_{0}^{\tau} F e^{A t} B B^{\prime} e^{A^{\prime} t} F^{\prime} d t \times \int_{0}^{\tau} e^{-2 r t \cos \theta} d t,
\end{aligned}
$$

(by Cauchy-Schwarz Inequality)

$$
\begin{aligned}
& =\|h(t)\|_{2}^{2} \times \frac{1-e^{-2 r \tau \cos \theta}}{2 r \cos \theta} \\
& \leq\|h(t)\|_{2}^{2} \tau<\infty, \quad \text { if } \theta \in[-\pi / 2, \pi / 2] .
\end{aligned}
$$

Thus, both $H(s)$ and $Y(s) \in H_{\infty}$.

To show that $N(s), D(s), X(s)$ and $Y(s)$ satisfy the Bezout identity (24), note that

$$
\begin{aligned}
Y(s)= & 1+F e^{-(s I-A) \tau}(s I-A+L C)^{-1} B \\
& +F\left(I-e^{-(s I-A) \tau}\right)(s I-A)^{-1} B \\
= & 1+F(s I-A)^{-1} B+F e^{-(s I-A) \tau} \\
& \times\left[(s I-A+L C)^{-1}-(s I-A)^{-1}\right] B \\
= & 1+F(s I-A)^{-1} B-F e^{-(s I-A) \tau}(s I-A+L C)^{-1} \\
& \times L C(s I-A)^{-1} B \\
= & 1+F(s I-A)^{-1} B-X(s) \frac{N(s)}{D(s)} .
\end{aligned}
$$

Hence,

$$
\begin{aligned}
D(s) Y(s)+N(s) X(s) & =D(s)\left[1+F(s I-A)^{-1} B\right] \\
& =\frac{1+F(s I-A)^{-1} B}{1+C(s I-A)^{-1} L}=1,
\end{aligned}
$$

which follows from noting that

(i) the zeros of $1+C(s I-A)^{-1} L$ are the eigenvalues of $A-L C$,

(ii) the zeros of $1+F(s I-A)^{-1} B$ are the eigenvalues of $A-B F$, which are the same as those of $A-L C$,

(iii) the poles of $1+C(s I-A)^{-1} L$ are the eigenvalues of $A$,

(iv) the poles of $1+F(s I-A)^{-1} B$ are the eigenvalues of $A$,

(v) $\lim _{s \rightarrow \infty} \frac{1+F(s I-A)^{-1} B}{1+C(s I-A)^{-1} L}=1$.

\subsection{Proof of Theorem 2.1}

We use the coprime factorisation $G(s)=N(s) / D(s)$ and $X(s)$ and $Y(s)$ from Lemma 3.1 with $F$ as in (11), and $L$ such that $(A-L C)$ has the same spectrum as $(A-B F)$. Without loss of generality, we assume from now on that $A$ has no eigenvalues on the $j \omega$-axis, so that $(A-B F)$ is Hurwitz as required in Lemma 3.1. ${ }^{4}$

From the above coprime factorisation, all stabilising LTI controllers for $G(s)$ are parametrised as

$$
K(s)=\frac{X(s)+D(s) Q(S)}{Y(s)-N(s) Q(s)},
$$

\footnotetext{
4 Otherwise, our arguments can be carried out in the same manner by taking $F_{\varepsilon}=B^{\prime} P_{\varepsilon}$, where $P_{\varepsilon}$ is the unique symmetric positive defi nite solution of the perturbed minimum energy Riccati equation $P_{\varepsilon} A+A^{\prime} P_{\varepsilon}=P_{\varepsilon} B B^{\prime} P_{\varepsilon}-\varepsilon^{2} I$, where $\varepsilon$ is a small parameter. The matrix $\left(A-B F_{\varepsilon}\right)$ will then be Hurwitz for all $\varepsilon>0$, and we would obtain (13) in the limit as $\varepsilon \rightarrow 0$.
}

where $Q(s)$ is an arbitrary transfer function in $H_{\infty}$, and the transfer function $T(s)$ between $n$ and $y$ in Figure 1 can be written as

$$
T(s)=1-D(s) Y(s)+D(s) N(s) Q(s) .
$$

Notice that because $F$ is the minimum energy state feedback gain, the spectrum of $(A-B F)$, and that of $(A-L C)$ by assumption, consists of the open loop stable eigenvalues of $A$ and the mirror image with respect to the $j \omega$-axis of the open loop unstable eigenvalues of $A$. Thus $D(s)$ is all pass and following similar steps to those in Middleton et al. (2004, Appendix A) it can be shown that

$$
\begin{aligned}
\inf _{Q \in H_{\infty}}\|T\|_{H_{2}}^{2}= & \sum_{k=1}^{m} 2 \operatorname{Re}\left\{p_{k}\right\}+\inf _{Q \in H_{\infty}}\|1-Y+N Q\|_{L_{2}}^{2} \\
= & \sum_{k=1}^{m} 2 \operatorname{Re}\left\{p_{k}\right\} \\
& +\inf _{Q \in H_{\infty}}\left\|e^{s \tau}(1-Y)+N_{0} Q\right\|_{L_{2}}^{2}
\end{aligned}
$$

Now, from (23), the term $e^{s \tau}(1-Y(s))$ in (29) is

$$
e^{s \tau}(1-Y(s))=\underbrace{-e^{s \tau} H(s)}_{\in H_{2}^{\perp}}-\underbrace{F e^{A \tau}(s I-A+L C)^{-1} B}_{\in H_{2}},
$$

where $H(s)$ is as defined in (26). Because the impulse response of $H(s)$ vanishes outside $[0, \tau]$, that of $e^{s \tau} H(s)$ vanishes outside $[-\tau, 0)$ and thus $e^{s \tau} H(s) \in$ $H_{2}^{\perp}$. On the other hand, $F e^{A \tau}(s I-A+L C)^{-1} B \in H_{2}$, since it is rational, strictly proper and stable. Hence, in (29),

$$
\begin{aligned}
\inf _{Q \in H_{\infty}} & \left\|e^{s \tau}(1-Y)+N_{0} Q\right\|_{L_{2}}^{2} \\
= & \left\|e^{s \tau} H(s)\right\|_{L_{2}}^{2} \\
& \quad+\inf _{Q \in H_{\infty}}\left\|-F e^{A \tau}(s I-A+L C)^{-1} B+N_{0} Q\right\|_{L_{2}}^{2} \\
= & \|H(s)\|_{L_{2}}^{2},
\end{aligned}
$$

(because $e^{s \tau}$ is all pass and $N_{0}(s)$ minimum phase)

$$
=\operatorname{trace} \int_{0}^{\tau} F e^{A t} B B^{\prime} e^{A^{\prime} t} F d t,
$$

where the last step follows from using Parseval's formula and the expression of the impulse response of $H(s)$ in (27), which concludes the proof.

\subsection{Proof of Corollary 2.2}

To prove Corollary 2.2 we will use (13) and the following fact.

Fact 1. (Rugh (1995, Exercise 7.12, p.127)). Given any two real matrices $A, M \in \mathbb{R}^{n \times n}$, if there exists a matrix $Q \in \mathbb{R}^{n \times n}$ such that $Q A^{\prime}+A Q=M$, then for all $\tau \geq 0$ $\int_{0}^{\tau} e^{A \sigma} M e^{A^{\prime} \sigma} d \sigma=e^{A \tau} Q e^{A^{\prime} \tau}-Q$.

\section{Proof of Corollary 2.2}

For technical simplicity suppose that $A$ has no eigenvalues on the $j \omega$-axis (see Footnote 4 ), and let $Q$ be 
the unique positive definite solution of the Lyapunov equation $Q A^{\prime}+A Q=B B^{\prime}$. Then, from Fact 1 ,

$$
\int_{0}^{\tau} F e^{A t} B B^{\prime} e^{A^{\prime} t} F^{\prime} d t=F\left(e^{A \tau} Q e^{A^{\prime} \tau}-Q\right) F^{\prime} .
$$

Let $F=B^{\prime} P$ as in Lemma 2.1. By replacing $F=B^{\prime} P$ in (30) we obtain

$$
\begin{aligned}
& \operatorname{trace}\left\{F\left(e^{A \tau} Q e^{A^{\prime} \tau}-Q\right) F^{\prime}\right\} \\
& =\operatorname{trace}\left\{B^{\prime} P\left(e^{A \tau} Q e^{A^{\prime} \tau}-Q\right) P B\right\}, \\
& =\operatorname{trace}\left\{P B B^{\prime} P\left(e^{A \tau} Q e^{A^{\prime} \tau}-Q\right)\right\}, \\
& =\operatorname{trace}\left\{\left(P A+A^{\prime} P\right)\left(e^{A \tau} Q e^{A^{\prime} \tau}-Q\right)\right\},
\end{aligned}
$$

since $P B B^{\prime} P=P A+A^{\prime} P$. Now, by expanding (31) and using the properties $A e^{A \tau}=e^{A \tau} A$, trace $\{M+N\}=$ $\operatorname{trace}\{M\}+\operatorname{trace}\{N\}$, and trace $\{M N\}=\operatorname{trace}\{N M\}$, and then collecting factors, we have

$$
\begin{aligned}
& \operatorname{trace}\left\{F\left(e^{A \tau} Q e^{A^{\prime} \tau}-Q\right) F^{\prime}\right\} \\
& =\operatorname{trace}\left\{\left(e^{A^{\prime} \tau} P e^{A \tau}-P\right)\left(A Q+Q A^{\prime}\right)\right\}, \\
& =\operatorname{trace}\left\{B^{\prime}\left(e^{A^{\prime} \tau} P e^{A \tau}-P\right) B\right\}, \text { since } A Q+Q A^{\prime}=B B^{\prime}, \\
& =\operatorname{trace}\left\{B_{1}^{\prime}\left(e^{A_{1}^{\prime} \tau} P_{1} e^{A_{1} \tau}-P_{1}\right) B_{1}\right\}, \text { from (10) and (11), } \\
& =\operatorname{trace}\left\{B_{1}^{\prime} P^{1 / 2}\left(e^{\Gamma^{\prime} \tau} e^{\Gamma \tau}\right) P_{1}^{1 / 2} B_{1}\right\}-B_{1}^{\prime} P_{1} B_{1},
\end{aligned}
$$

where $\Gamma=P^{1 / 2} A P^{-1 / 2}$.

Note that in general $e^{\Gamma^{\prime} \tau} e^{\Gamma \tau} \neq e^{\left(\Gamma^{\prime}+\Gamma\right) \tau}$, and it can be shown that equality holds if and only iff $\Gamma \Gamma^{\prime}-\Gamma^{\prime} \Gamma=0$. Otherwise, a result due to Cohen (1988) states that

$$
\lambda_{\max }\left(e^{\Gamma^{\prime} \tau} e^{\Gamma \tau}\right) \leq \lambda_{\max }\left(e^{\left(\Gamma^{\prime}+\Gamma\right) \tau}\right),
$$

where $\lambda_{\max }(\cdot)$ denotes the maximum eigenvalue. The inequality (33) and the fact that

$$
\begin{aligned}
\operatorname{trace}\left\{\Gamma^{\prime}+\Gamma\right\} & =\operatorname{trace}\left\{P_{1}^{-1 / 2} A^{\prime} P_{1}^{1 / 2}+P_{1}^{1 / 2} A P_{1}^{-1 / 2}\right\} \\
& =B_{1}^{\prime} P_{1} B_{1}=\sum_{k=1}^{m} 2 \operatorname{Re}\left\{p_{k}\right\},
\end{aligned}
$$

in (32) yield (17). The $O\left(\tau^{2}\right)$ equality in Corollary 2.2 follows from (32) and the fact that

$$
e^{\left(\Gamma^{\prime}+\Gamma\right) \tau}-e^{\Gamma^{\prime} \tau} e^{\Gamma \tau}=O\left(\tau^{2}\right)\left(\Gamma \Gamma^{\prime}-\Gamma^{\prime} \Gamma\right),
$$

as may be verified by a Taylor expansion (Bellman, 1970, p. 173).

\section{CONCLUSIONS}

This paper has extended the bound from Braslavsky et al. (2004) on the lowest SNR required for feedback stabilisation over a SNR constrained communication channel to plants with a time delay. An exact expression for the additional penalty introduced by the time delay was given, and also a bound showing the explicit dependence on unstable poles. The bound grows exponentially on the time delay and the unstable poles of the system. This bound is less conservative for small time delays, and otherwise, a nonconservative numerical estimate cost could be computed by approximating the delay with a Padé approximant and using the formula for the critical SNR derived for nonminimum phase plants in Middleton et al. (2004).

\section{REFERENCES}

R. Bellman. Introduction to matrix analysis. McGrawHill, 1970.

J.H. Braslavsky, R.H. Middleton, and J.S. Freudenberg. Feedback stabilisation over signal-to-noise ratio constrained channels. In Proc. 2004 American Control Conference, Boston, USA, July 2004.

J.E. Cohen. Spectral inequalities for matrix exponentials. Linear Algebra and its Applications, 111:2528, December 1988.

T.M. Cover and J.A. Thomas. Elements of Information Theory. John Wiley \& Sons, 1991.

D.F. Delchamps. Stabilizing a linear system with quantized state feedback. IEEE Trans. on Automatic Control, 35(8):916-924, August 1990.

N. Elia and S.K. Mitter. Stabilization of linear systems with limited information. IEEE Trans. on Automatic Control, 46(9):1384-1400, September 2001.

G.H. Golub and C.F. Van Loan. Matrix Computations. The John Hopkins University Press, 3rd edition, 1996.

H. Ishii and B.A. Francis. Quadratic stabilization of sampled-data systems with quantization. Automatica, 39(10):1793-1800, 2003.

Feng-Li Lian, J. Moyne, and D. Tilbury. Network design consideration for distributed control systems. IEEE Transactions on Control Systems Technology, 10(2):297-307, March 2002.

D. Liberzon. On stabilization of linear systems with limited information. IEEE Trans. on Automatic Control, 48(2):304-307, February 2003.

R.H. Middleton, J.H. Braslavsky, and J.S. Freudenberg. Stabilization of non-minimum phase plants over signal-to-noise ratio constrained channels. In Proc. 5th Asian Control Conference, Melbourne, Australia, July 2004.

L. Mirkin and N. Raskin. Every stabilizing dead-time controller has an observer-predictor-based structure. Automatica, 39:1747-1754, 2003.

G.N. Nair and R.J. Evans. Mean square stabilisability of stochastic linear systems with data rate constraints. In Proc. 41st IEEE CDC, pages 16321637, Las Vegas, Nevada, USA, December 2002.

G.N. Nair, R.J. Evans, I.M.Y. Mareels, and W. Moran. Topological feedback entropy for nonlinear systems. In Proc. of the 2004 Asian Control Conference, pages 1933-1940, Melbourne, Australia, July 2004.

Wilson J. Rugh. Linear System Theory. Prentice Hall, 2nd edition, 1995.

W.S. Wong and R.W. Brockett. Systems with finite communication bandwidth constraints. ii. stabilization with limited information feedback. IEEE Trans. on Automatic Control, 44(5):1049-1053, May 1999. 